\title{
Join Decompositions for Efficient Synchronization of CRDTs after a Network Partition
}

\author{
[Work in progress report] \\ Vitor Enes* \\ FCT, Universidade Nova de Lisboa \\ Lisboa, Portugal \\ Carlos Baquero; Paulo Sérgio Almeidał \\ Ali Shoker ${ }^{\dagger}$ \\ HASLab / INESC TEC \& Universidade do Minho \\ Braga, Portugal
}

\begin{abstract}
State-based CRDTs allow updates on local replicas without remote synchronization. Once these updates are propagated, possible conflicts are resolved deterministically across all replicas. $\delta$-CRDTs bring significant advantages in terms of the size of messages exchanged between replicas during normal operation. However, when a replica joins the system after a network partition, it needs to receive the updates it missed and propagate the ones performed locally. Current systems solve this by exchanging the full state bidirectionally or by storing additional metadata along the CRDT. We introduce the concept of join-decomposition for statebased CRDTs, a technique orthogonal and complementary to delta-mutation, and propose two synchronization methods that reduce the amount of information exchanged, with no need to modify current CRDT definitions.
\end{abstract}

\section{CCS Concepts}

-Theory of computation $\rightarrow$ Distributed algorithms;

\section{Keywords}

State Synchronization, Replication, CRDTs.

\begin{abstract}
*European Union Seventh Framework Program (FP7/20072013) under grant agreement 609551, SyncFree project.

†Project "TEC4Growth - Pervasive Intelligence, Enhancers and Proofs of Concept with Industrial Impact/NORTE-010145-FEDER-000020" is financed by the North Portugal Regional Operational Programme (NORTE 2020), under the PORTUGAL 2020 Partnership Agreement, and through the European Regional Development Fund (ERDF).
\end{abstract}

Permission to make digital or hard copies of all or part of this work for personal or classroom use is granted without fee provided that copies are not made or distributed for profit or commercial advantage and that copies bear this notice and the full citation on the first page. Copyrights for components of this work owned by others than the author(s) must be honored. Abstracting with credit is permitted. To copy otherwise, or republish, to post on servers or to redistribute to lists, requires prior specific permission and/or a fee. Request permissions from permissions@ acm.org.

PMLDC '16, July 17 2016, Rome, Italy

(C) 2016 Copyright held by the owner/author(s). Publication rights licensed to ACM ISBN 978-1-4503-4775-4/16/07 . \$ \$15.00

DOI: http://dx.doi.org/10.1145/2957319.2957374

\section{INTRODUCTION}

The concept of Conflict-free Replicated Data Type (CRDT) was introduced in [7] and presents two flavors of CRDTs: state-based and operation-based. A state-based CRDT can be defined as a triple $(S, \sqsubseteq, \sqcup)$ where $S$ is a join-semilattice, $\sqsubseteq$ its partial order, and $\sqcup$ is a binary join operator that derives the least upper bound for every two elements of $S$.

With $\delta$-CRDTs $[1,2]$, every time a replica performs an update, it will only send the information needed to reflect this update in other replicas, with the anti-entropy algorithm keeping at each node metadata tracking which deltas still need to be propagated to current peers. However, after a long partition, such metadata is discarded. In this situation, when a replica goes online again, the other remote replicas typically send their full state so this replica sees the updates it missed.

The work presented in [6] introduces the concept of $\Delta$ CRDTs where replicas exchange metadata used to calculate a $\Delta$ that reflects the missed updates. As this metadata is typically smaller than the full state, less is demanded from the network. In this approach CRDTs need to be extended to maintain the additional metadata for $\Delta$ derivation, and if this metadata needs to be garbage collected the mechanism will fall-back to standard full state transmission.

In this paper we will present a mechanism that does not add additional metadata to standard state-based CRDTs, but instead is able to decompose the state into smaller states than can be selected and grouped in a $\Delta$ for efficient transmission.

\subsection{Problem Statement}

Consider replica $A$ with state $a$ and replica $B$ with state $b$, which at some point stop disseminating updates but keep updating their local state. When these replicas go online, what should replica $A$ send to replica $B$ so that $B$ sees the updates performed on $a$ since they stopped communicating? We could try to find $c$ such that:

$$
a=b \sqcup c
$$

but if both replicas performed updates while they were offline, their states are concurrent, and there's no such $c$. (We say two states $a$ and $b$ are concurrent if $a$ is not less than $b$ and $b$ is not less than $a$ in the partial order: $a \| b \Longleftrightarrow a \nsubseteq$ $b \wedge b \nsubseteq a$.) The trick is how to find $c$ ( $\Delta$ from now on) which reflects the updates in the join of $a$ and $b$ still missing in $b$ :

$$
a \sqcup b=b \sqcup \Delta
$$


The trivial example would be $\Delta=a$, but we would like to send less information than the full state. So, how can replica $A$ calculate a smaller $\Delta$ to be sent to replica $B$, reflecting the missed updates?

\subsection{Contributions}

Firstly, we introduce the concept of join-decomposition for state-based CRDTs, a technique orthogonal and complementary to delta-mutation. Then, we propose two synchronization techniques. State Driven: replica $B$ sends its full state $b$ to replica $A$ and replica $A$ is able to derive $\Delta$. Digest Driven: replica $B$ sends some information about its state $b$, smaller than $b$ itself, but enough to allow replica $A$ to compute $\Delta$.

\section{JOIN DECOMPOSITIONS}

We now explain how the concept of join-decomposition [4] can be applied to state-based CRDTs. Given state $r \in S$, we say that $D \in \mathcal{P}(S)$ is a join-decomposition of $r$ if:

$$
\begin{gathered}
\bigsqcup D=r \\
\forall s \in D \cdot \bigsqcup(D \backslash\{s\}) \sqsubset r
\end{gathered}
$$

Property (i) states that the join of all elements in a joindecomposition of $r$ should be $r$. Property (ii) says that each element in a join-decomposition is not redundant: joining the remaining elements is not enough to produce $r$.

We are interested in decompositions made up of "basic" irreducible elements. An element $s$ is join-irreducible if it cannot result from a join of two elements other than itself, i.e.:

$$
t \sqcup u=s \Rightarrow t=s \vee u=s
$$

We say $D$ is a join-irreducible decomposition if $D$ is a join-decomposition and:

$$
\forall s \in D \cdot s \text { is join-irreducible }
$$

States in common CRDTs typically have join-irreducible decompositions, and we now present some examples of decomposition functions, which take a state and return a joinirreducible decomposition.

\subsection{Example Decompositions}

A GCounter is a simple replicated counter where its value can only increase [2]. It is represented as a map from ids to naturals, i.e., GCounter $=\mathbb{I} \hookrightarrow \mathbb{N}$, and each replica can only increase the value of the counter in its position of the map. The value of the counter is the sum of all increments. For example, $p=\{A \mapsto 3, B \mapsto 5\}$ means replica $A$ has incremented the counter three times, replica $B$ five times, hence the value is eight. For each state $s$, a join-irreducible decomposition can be obtained by function:

$$
\mathrm{D}^{\mathrm{GCounter}}(s)=\{\{i \mapsto v\} \mid(i, v) \in s\}
$$

The decomposition for the GCounter $p$ above would be $\{\{A \mapsto 3\},\{B \mapsto 5\}\}$.

To allow both increments and decrements we can compose two GCounter by pairing them [3] and we have a PNCounter =
$(\mathbb{I} \hookrightarrow \mathbb{N}) \times(\mathbb{I} \hookrightarrow \mathbb{N})$. Join-irreducible decompositions can be obtained through:

$$
\begin{aligned}
\mathrm{D}^{\mathrm{PNC} C o u n t e r}((p, n)) & =\{(\{i \mapsto v\},\{\}) \mid(i, v) \in p\} \\
& \cup\{(\{\},\{i \mapsto v\}) \mid(i, v) \in n\}
\end{aligned}
$$

As a final example, an Add-Wins set has state AWSet $=$ $(E \hookrightarrow \mathcal{P}(D)) \times \mathcal{P}(D)$. This CRDT is a pair where the first component is a map (from element, in $E$, to a set of supporting dots (unique event identifiers), in $\mathcal{P}(D)$ ) and the second component is a causal context represented as a set of $\operatorname{dots} \mathcal{P}(D)$ [2]. When an element is added to the set, a new entry in the map is created, if needed, mapping this element to a new dot, and current dots for the element, if any, are discarded. This new dot is also added to the causal context. To remove an element, we remove its entry from the map. An example for this data type where two elements ( $x$ and $y$ ) were added and another (initially marked with unique dot $a 2)$ was removed is $s=(\{x \mapsto$ $\{a 1\}, y \mapsto\{b 1, c 1\}\},\{a 1, a 2, b 1, c 1\})$. (The range function rng returns all sets of supporting dots in the mapping.) The join-irreducible decomposition of state $(m, c)$ can be obtained through function:

$$
\begin{aligned}
\mathrm{D}^{\mathrm{AWSet}}((m, c)) & =\{(\{e \mapsto\{d\}\},\{d\}) \mid(e, s) \in m, d \in s\} \\
& \cup\{(\{\},\{d\}) \mid d \in c \backslash \bigcup \mathrm{rng} m\}
\end{aligned}
$$

The join-irreducible decomposition for the state $s$ above is:

$$
\begin{aligned}
& \{(\{x \mapsto\{a 1\}\},\{a 1\}), \\
& (\{y \mapsto\{b 1\}\},\{b 1\}), \\
& (\{y \mapsto\{c 1\}\},\{c 1\}), \\
& (\{\},\{a 2\})\}
\end{aligned}
$$

\section{EFFICIENT SYNCHRONIZATION}

\section{State Driven.}

The State Driven approach can be applied to all statebased CRDTs as long as we have a corresponding joindecomposition. We define $\min ^{\Delta}: S \times S \rightarrow S$ as a function that given two states (the local state $a$ and the remote replica state $b$ ) will produce a $\Delta$. Join-irreducible decompositions will in general produce smaller $\Delta$ s. Let D $: S \rightarrow \mathcal{P}(S)$ be a function that produces a join-decomposition.

$$
\min ^{\Delta}(a, b)=\bigsqcup\{s \mid s \in \mathrm{D}(a) \wedge b \sqsubset b \sqcup s\}
$$

This $\min ^{\Delta}$ function joins all $s$ in the local state joindecomposition that strictly inflate the remote state. If the local replica ships the resulting $\Delta$, to be joined to the remote replica, and joins the state received from the remote replica to its local state, both these replicas will reach convergence (if in the meantime no new update was performed).

\section{Digest Driven.}

With the Digest Driven approach we achieve the same results of State Driven but by exchanging less information. We re-define $\min ^{\Delta}: S \times M \rightarrow S$ as a function that given the local state $a$ and some digest $m$ related to the remote state will produce a $\Delta$.

$$
\min ^{\Delta}(a, m)=\bigsqcup\{s \mid s \in \mathrm{D}(a) \wedge \inf (s, m)\}
$$


This digest will be data-type specific, which means that $\min ^{\Delta}$ will use a type-specific function $\inf (s, m)$ to check if $s$ inflates the remote state summarized by the received digest $m$.

A digest extraction function digest : $S \rightarrow M$ and the inflation test inf : $S \times M \rightarrow \mathbb{B}$ for the causal AWSet CRDT can be defined as:

$$
\begin{aligned}
\text { digest }^{\mathrm{AWSet}}((m, c)) & =(\bigcup \mathrm{rng} m, c) \\
\inf ^{\mathrm{AWSet}}((e,\{d\}),(a, c)) & = \begin{cases}T & \text { if } d \notin c \vee(e=\{\} \wedge d \in a) \\
F & \text { otherwise }\end{cases}
\end{aligned}
$$

The function digest ${ }^{\text {AWSet }}$ returns a pair where the first component is the set of active dots (the supporting dots of elements that were added and not yet removed) and the second component is the full causal context. The inflation check inf $^{\text {AWSet }}$ will return $T$ for $s \in \mathrm{D}(a)$ if the $\operatorname{dot}$ in $s$ has not been seen in the other replica or $s$ represents a removed element (i.e., $(\{\},\{d\}))$ that has been added and not yet removed in the other replica ( $d$ is still in the active dots).

If the Digest Driven technique is performed bidirectionally and no updates occurred, both replicas will converge (otherwise, they can still be collected separately in a dedicated buffer for further transmission).

\section{FINAL REMARKS}

We explain how the concept of join-decomposition can be applied in two different synchronization techniques which do not require extending current CRDTs implementations as proposed by [6]. Further research is needed to understand the trade-offs between both approaches in terms of storage and network consumption.

Reference implementations of join-decompositions are publicly available in GitHub [5].

\section{REFERENCES}

[1] P. S. Almeida, A. Shoker, and C. Baquero. Efficient State-Based CRDTs by Delta-Mutation. In Networked Systems - Third International Conference, NETYS 2015, Agadir, Morocco, May 13-15, 2015, pages 62-76.

[2] P. S. Almeida, A. Shoker, and C. Baquero. Delta State Replicated Data Types. CoRR, abs/1603.01529, 2016.

[3] C. Baquero, P. S. Almeida, A. Cunha, and C. Ferreira. Composition of State-based CRDTs. 2015.

[4] G. Birkhoff. Rings of sets. Duke Math. J., 3(3):443-454, 1937.

[5] Lasp. Types. URL http://github.com/lasp-lang/types.

[6] A. Linde, J. Leitão, and N. Preguiça. $\Delta$-CRDTs: Making $\delta$-CRDTs Delta-Based. PaPoc 2016, 2016.

[7] M. Shapiro, N. Preguiça, C. Baquero, and M. Zawirski. Conflict-free Replicated Data Types. Technical Report RR-7687, July 2011. 